\title{
Synthesis and Antimicrobial Evaluation of Some New Benzoxazinone and Quinazolinone Derivatives
}

\author{
Assy, M.G. ${ }^{(a)}$, Abdalha, A.A. ${ }^{*}(b)$, Abdel Aziz, A.E ${ }^{(c)}$
}

(a) Chemistry Department, Faculty of Science, Zagazige University.

b) Chemistry Department, Faculty of Science, Ain shams University, Abassia, Cairo, Egypt, Current address, Chemistry Department, Faculty of Science, Tabuk University, KSA.

$$
\text { E - mail: abdoelaal@yahoo.com }
$$

c)Chemistry Department, Faculty of Science, Zagazige University, Current address, Chemistry Department, Faculty of Science, Tabuk University, KSA.

${ }^{*}$ Corresponding author: Abdalha, A.A., Chemistry Department. Faculty of science, Tabuk University, KSA.

$$
\text { E - mail: abdoelaal@yahoo.com }
$$

\section{ABSTRACT}

Ring opening reactions of benzoxazinone (2) with nitrogen and oxygen nucleophiles gave the corresponding benzamides (3)\&(4) and benzoates (5).Quinazolinone derivatives (6) and (7) were obtained upon treatment of (2) with hydrazine hydrate and/or hydroxylamine hydrochloride.Triazole derivatives $(8 a-b)$ were obtained when (2) was subjected to react with semicarbazide and/or thiosemicarbazide respectively. Reaction of (2) with sodium azide gave a mixture of tetrazole derivative (9) and benzoimidazolone derivative (10). The antimicrobial activity of some synthesized compounds was examined against bacteria, fungi and yeast. Some of the tested compounds showed promising activities.

\section{KEY WORDS}

Benzoxazinone; Quinazolinone; Benzoimidazolone; Triazole; Tetrazole.

\section{Council for Innovative Research}

\section{Journal: Journal of Advances in Chemistry}

Peer Review Research Publishing System

Vol. 8, No. 1

editor@cirworld.com 


\section{INTRODUCTION}

The reported biological and pharmacological activities of benzoxazinone and quinazolinone derivatives, such as anticonvulsant [1-3], antihistaminic [4-5], antihypertensive [6-8], analgesic [9-10], anti-inflammatory [11], antimicrobial [12-15] and antifungal [16-17] have stimulated us to synthesize some new derivatives of these classes of compounds with the hope of obtaining new structures with enhanced potency or finding new applications. Also we aimed to incorporate a sterically bulky group such as phenyl group in position-2 to detect its role in the nature and ease of reaction of these compounds.

\subsection{RESULTS AND DISCUSSION}

In continuation of our efforts to develop the synthesis and reactivity of benzoxazinone derivatives toward some nitrogen and oxygen nucleophilic reagents [8,18-21], we reported herein, the synthesis of novel 2-phenyl-6- iodo-3,1benzoxazin-4-one (2) via condensation of benzoyl chloride with 5- iodoanthranilic acid in prydine via the intermediacy of benzoic acid derivative (1)(scheme1). The structures of $(\mathbf{1 , 2})$ have been confirmed by their analytical data as well as spectroscopic data (IR, ${ }^{3}-\mathrm{NMR}$ and MS).Ring opening of (2) with primary amines, such as 4methoxyaniline,benzylamine,ethylamine,methylamine and sec.butylamine beside hydrazinolysis with hydrazine hydrate and phenyl hydrazine gave the corresponding benzamide derivatives (3a-g) respectively (scheme 2). The structures of $(3 \mathbf{a}-\mathbf{g})$ have been confirmed by their analytical data as well as spectroscopic data(IR, ${ }^{1} \mathrm{H}-\mathrm{NMR}$ and MS). With secondary amines, such as morpholine, piperidine and /or piperazine in 1,4-dioxane, benzoxazinone(2) yielded the expected products $(\mathbf{4 a - c )}$ respectively (scheme 2$)$. The structures of $(\mathbf{4 a - c )}$ have been confirmed by their analytical data as well as spectroscopic data (IR, ${ }^{1} \mathrm{H}-\mathrm{NMR}$ and MS).. However, when the reaction was carried out in ethanol and/or $n-$ butanol the ester products $(5 \mathbf{a}-\mathbf{b})($ scheme 2 )were obtained in fairly good yield, which means that morpholine, piperidine and/or piperazine act as a base favoring the formation of alkoxide ion which promotes ring opening of (2) to give the esters (5ab); this observation was supported by the reaction of (2) with ethanol and/or $n$-butanol in the presence of pyridine as a base to give the esters $(5 a-b)($ scheme 2$)$. The structures of $(5 a-b)$ have been confirmed by their analytical data as well as spectroscopic data (IR, ${ }^{1} \mathrm{H}-\mathrm{NMR}$ and MS).

On the other hand, hydrazinolysis of (2) with hydrazine hydrate or reaction with hydroxylamine hydrochloride in butanol gave the corresponding quinazolinone derivatives $(6 \mathbf{a}-\mathbf{b})$ respectively (scheme 2$)$, whose structures were confirmed by elemental analysis as well as spectroscopic data (IR, ${ }^{1} \mathrm{H}-\mathrm{NMR}$ and MS).

Furthermore, fusion of (2) with ammonium acetate (at ca. $170^{\circ} \mathrm{C}$ ) gave the quinazolinone derivative (7)(scheme 3), whose structure was established by elemental analysis as well as spectroscopic data (IR, ${ }^{1} \mathrm{H}-\mathrm{NMR}$ and MS).

Moreover, refluxing of (2) with semicarbazide and/or thiosemicarbazide in pyridine yielded the triazole derivatives $(\mathbf{8} \mathbf{a}-\mathbf{b})$ respectively (scheme 3$)$. The structures of $(\mathbf{8 a - b})$ have been confirmed by their analytical data as well as spectroscopic data (IR, ${ }^{1} \mathrm{H}-\mathrm{NMR}$ and MS).

Reaction of benzoxazinone(2) with sodium azide in acetic acid afforded a mixture of tetrazole derivative (9) and benzoimidazolone derivative (10)(scheme 3 ) according to the following pathway:-

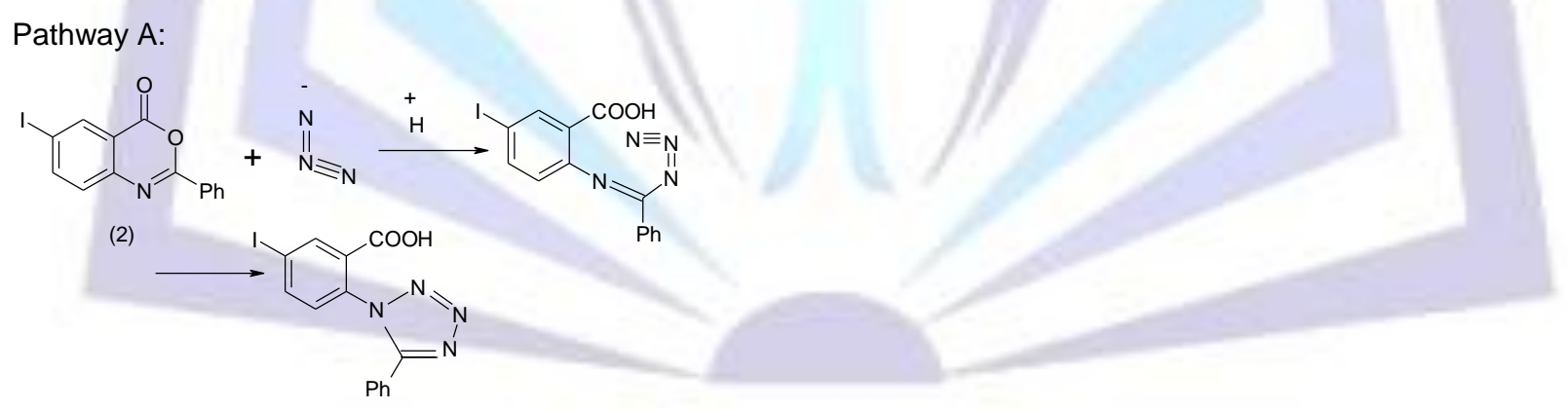

(9)

Pathway B: 


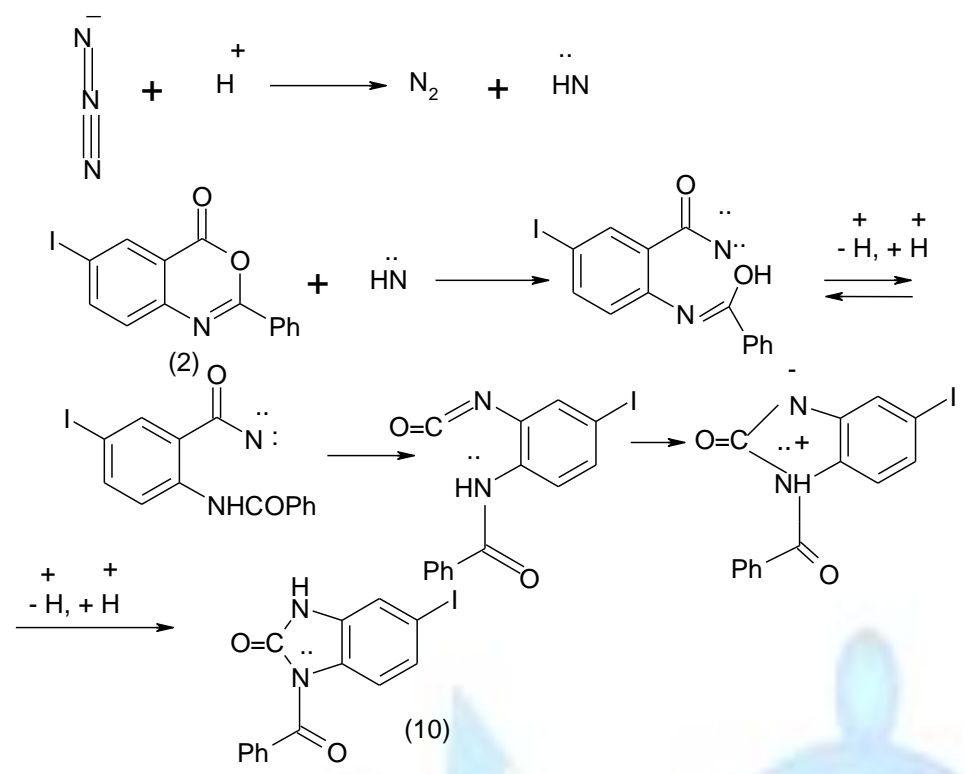

The structures of $(\mathbf{9 \&} \mathbf{1 0})$ were elucidated by elemental analysis as well as spectral data(IR, ${ }^{1} \mathrm{H}-\mathrm{NMR}$ and MS).

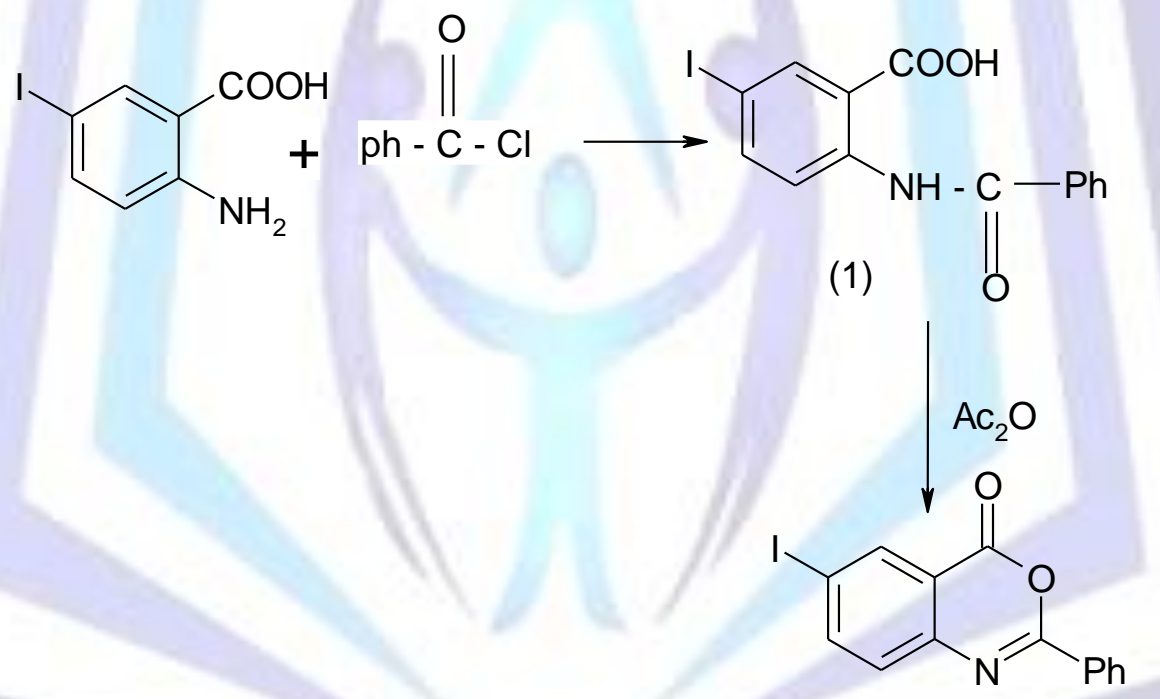

scheme 1

(2) 


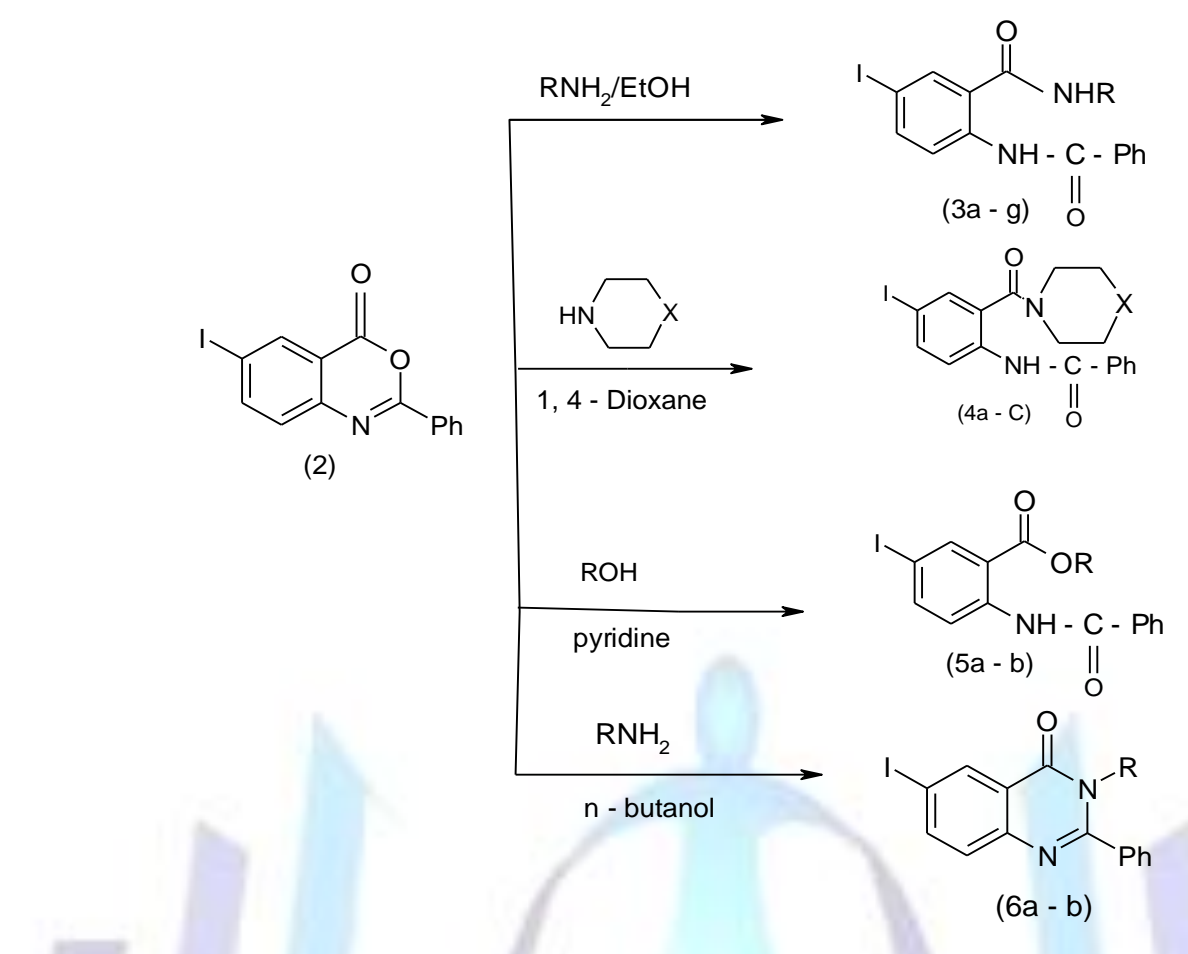

Scheme 2

3a) $\mathrm{R}=-\mathrm{C}_{6} \mathrm{H}_{4}\left(4-\mathrm{OCH}_{3}\right)$, 3b) $\left.\left.\left.\left.\mathrm{R}=-\mathrm{CH}_{2} \mathrm{Ph}, 3 \mathrm{c}\right) \mathrm{R}=-\mathrm{CH}_{2} \mathrm{CH}_{3}, 3 \mathrm{~d}\right) \mathrm{R}=-\mathrm{CH}_{3}, 3 \mathrm{e}\right) \mathrm{R}=-\mathrm{CH}\left(\mathrm{CH}_{3}\right) \mathrm{CH}_{2} \mathrm{CH}_{3}, 3 f\right) \mathrm{R}=-\mathrm{NH}_{2}$, 3g) $\mathrm{R}=-\mathrm{NHPh}$, 4a) $\mathrm{X}=\mathrm{O}$, 4b) $\left.\left.\mathrm{X}=\mathrm{CH}_{2}, 4 \mathrm{c}\right) \mathrm{X}=\mathrm{NH}, 5 \mathrm{a}\right) \mathrm{R}=-\mathrm{C}_{2} \mathrm{H}_{5}$,

5b) $\left.\left.\mathrm{R}=\mathrm{n}-\mathrm{C}_{4} \mathrm{H}_{9}, 6 \mathrm{a}\right) \mathrm{R}=-\mathrm{NH}_{2}, 6 \mathrm{~b}\right) \mathrm{R}=-\mathrm{OH}$.

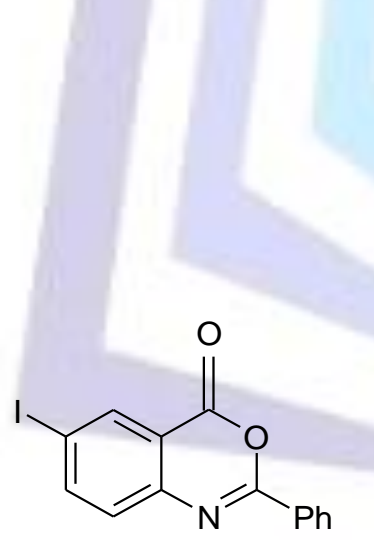

(2)

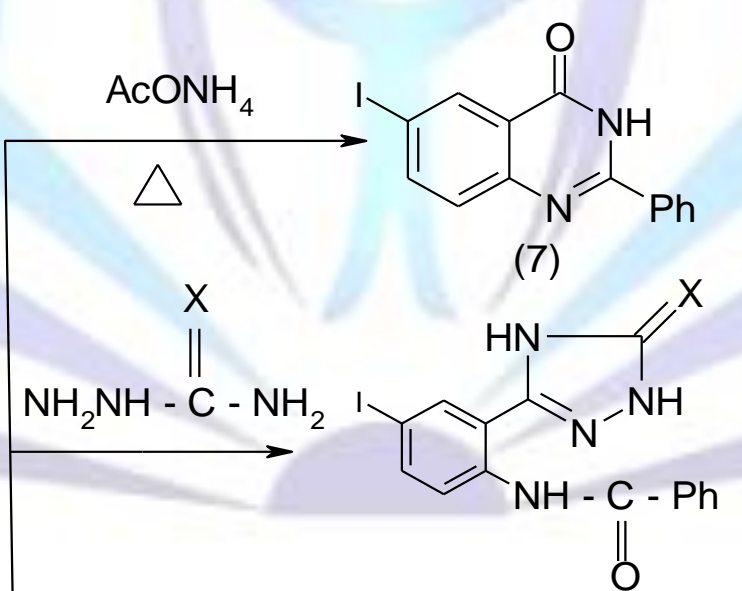

$(8 a-b)$

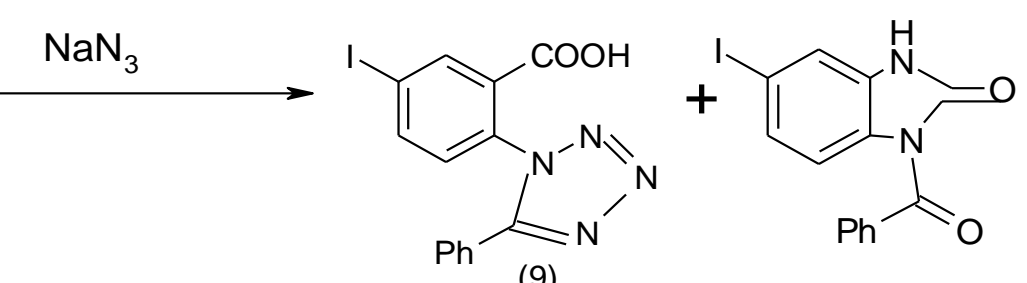

(9)

Scheme 3

8a) $X=O, 8 b) X=S$. 


\subsection{Antibacterial, Antifungal and Antiyeast Activation of the Synthesized Compounds}

The antimicrobial activities of the synthesized compounds were determined in vitro using the holeplate and filter paper disc method (Rosen, 1989) which considered the most commonly used technique for determining sensitivity of chemotherapeutic agents. Compounds were dissolved in $10 \%$ acetone at different concentrations $(125,250,500 \mu \mathrm{g} / \mathrm{ml})$. Agar plates were inoculated uniformly from fresh broth culture of Gram -ve bacteria (Escherichia coli), Gram +ve bacteria (Staphylococcus aureus), fungi (Aspergillus flavus), and yeast (Candida albicans). The disks were Incubated at $28^{\circ} \mathrm{C}$ for $24 \mathrm{hr}$, and the formed inhibition zones were diffused into the agar from the disk (this refers to the organism was inhibited by material) and were measured in $\mathrm{mm}$ [22-23].

Bacterial media: Nutrient agar and broth $(\mathrm{pH} 7.0)$, Peptone $(0.5 \mathrm{~g})$, Beef extract $(0.3 \mathrm{~g})$, Agar (15.0g) and distilled water $(1000.0 \mathrm{ml})$.

Fungal media: $\mathrm{MgSO}_{4}(0.5 \mathrm{~g}) ; \mathrm{KCl}(0.5 \mathrm{~g})$; Sucrose (30.0g); $\mathrm{FeSO}_{4}(0.01 \mathrm{~g}) ; \mathrm{NaNO}_{3}(3.0 \mathrm{~g}) ; \mathrm{K}_{2} \mathrm{HPO} 4$ (1.0g); Agar (15.0g) and distilled water $(1000.0 \mathrm{ml})$.

Table 1: Antimicrobial activity of some synthesized compounds.

\begin{tabular}{|c|c|c|c|c|c|c|c|c|}
\hline \multirow{3}{*}{$\begin{array}{c}\text { compoun } \\
\text { d }\end{array}$} & \multicolumn{4}{|c|}{ Bacteria } & \multicolumn{2}{|c|}{ Fungi } & \multicolumn{2}{|c|}{ Yeast } \\
\hline & \multicolumn{2}{|c|}{$\begin{array}{l}\text { Echerichi } \\
\left.\text { a coli ( } \mathbf{G}^{-}\right)\end{array}$} & \multicolumn{2}{|c|}{$\begin{array}{l}\text { Staphylococc } \\
\text { us aureus ( } \mathrm{G} \\
+ \text { ) }\end{array}$} & \multicolumn{2}{|c|}{$\begin{array}{l}\text { Aspergillu } \\
\text { s flavus } \\
\text { (Fungus) }\end{array}$} & \multicolumn{2}{|c|}{$\begin{array}{l}\text { Candida } \\
\text { albicans }\end{array}$} \\
\hline & A & MIC & A & MIC & A & MIC & A & MIC \\
\hline 1 & 26 & 125 & 34 & 250 & 0.0 & 125 & 0.0 & 125 \\
\hline 2 & 0.0 & 125 & 0.0 & 250 & 0.0 & 125 & 0.0 & 125 \\
\hline $3 a$ & 0.0 & 500 & 0.0 & 500 & 0.0 & 250 & 0.0 & 250 \\
\hline $3 \mathbf{b}$ & 0.0 & 125 & 0.0 & 125 & 0.0 & 250 & 0.0 & 500 \\
\hline $3 c$ & 0.0 & 250 & 0.0 & 125 & 0.0 & 250 & 0.0 & 250 \\
\hline $3 d$ & 0.0 & 125 & 0.0 & 125 & 0.0 & 250 & 0.0 & 250 \\
\hline $3 e$ & 0.0 & 125 & 0.0 & 250 & 0.0 & 125 & 0.0 & 125 \\
\hline $3 f$ & 0.0 & 125 & 0.0 & 125 & 0.0 & 25 & 0.0 & 125 \\
\hline $4 a$ & 0.0 & 125 & 0.0 & 250 & 0.0 & 125 & 0.0 & 125 \\
\hline $4 b$ & 0.0 & 500 & 0.0 & 250 & 0.0 & 125 & 0.0 & 250 \\
\hline $5 a$ & 25 & 250 & 33 & 250 & 0.0 & 125 & 13 & 250 \\
\hline $5 b$ & 39 & 250 & 43 & 125 & 0.0 & 125 & 0.0 & 250 \\
\hline $6 a$ & 10 & 250 & 11 & 125 & 0.0 & 250 & 0.0 & 250 \\
\hline 7 & 9 & 250 & 9 & 500 & 0.0 & 250 & 0.0 & 500 \\
\hline
\end{tabular}

$A=$ Antimicrobial activity of tested compounds

MIC = Minimum inhibitory concentration

\section{Conclusion}

Based on the results of the inhibition zoon and Minimum inhibitory concentration (MIC) $(\mu \mathrm{g} / \mathrm{ml})$ adata in table (1) revealed that aminoquinazolinon(6a) and quinazolinone (7) showed promising antimicrobial activity while benzamide derivative(1) and ester derivatives( $5 a)$ \&(5b) exhibited high antibacterial and antifungal activities. Compounds 2,(3a-f) and (4a-b) exhibited no antimicrobial activity. 


\section{Experimental}

Melting points were measured on an electro thermal melting point apparatus. Elemental analyses were carried out at the micro analytical unit, Cairo University, Giza, Egypt. The IR spectra were measured on a Unicam SP-1200 spectrometer using $\mathrm{KBr}$ Wafer technique. The ${ }^{1} \mathrm{H}-\mathrm{NMR}$ spectra were measured in DMSO- $\mathrm{d}_{6}$ on a Varian plus instrument $(300 \mathrm{MHz})$. Mass spectra were recorded on a shimadzu GC-MS QP- 1000EX instrument operating at 70 ev. Antimicrobial activity was carried out at micro analytical center, Faculty of science, Cairo University, Cairo, Egypt.

\subsection{2-Benzamido-5 -iodobenzoic acid (1)}

To a solution of 5 - iodoanthranilic acid $(0.01 \mathrm{~mol})$ in anhydrous pyridine $(50 \mathrm{~mL})$, benzoyl chloride $(0.01 \mathrm{~mol})$ was added dropwise at room temperature with stirring for $30 \mathrm{~min}$; then the reaction mixture was heated on a water bath for $5 \mathrm{hr}$. After cooling, the reaction mixture was poured on ice cold hydrochloric acid. The produced mass was filtered off, washed with water, dried and recrystallized from benzene to give (1).

Colour: brown, M.P.: 272- $273^{\circ} \mathrm{C}$, Yield: $60 \%$, IR $(\mathrm{KBr})\left(\mathrm{v}, \mathrm{cm}^{-1}\right): 3115(\mathrm{NH}), 1700,1658(\mathrm{C}=\mathrm{O}),{ }^{1} \mathrm{H}-\mathrm{NMR}\left(\mathrm{DMSO}-\mathrm{d}_{6}\right): \delta$ (ppm): $12.09(\mathrm{~s}, 1 \mathrm{H}, \mathrm{OH}), 8.53(\mathrm{~s}, 1 \mathrm{H}, \mathrm{NH}), 8.29(\mathrm{~s}, 1 \mathrm{H}, \mathrm{ArH}), 8.01-7.95(\mathrm{~m}, 3 \mathrm{H}, \mathrm{ArH}), 7.66-7.57(\mathrm{~m}, 3 \mathrm{H}, \mathrm{ArH}), 7.36(\mathrm{~s}$, $1 \mathrm{H}, \mathrm{ArH}) . \mathrm{MS}, \mathrm{m} / \mathrm{z}(\%): 367$ (M+1 100$), 245$ (100), 236 (50), 105 (100), 78 (95).

Anal.Calcd. For $\mathrm{C}_{14} \mathrm{H}_{10} \mathrm{NO}_{3} \mathrm{I}$ (367.14): C, 45.80, H, 2.74; N, 3.81. Found: C, 46.01, H, 2.77; N,4.02.

\subsection{6-lodo- 2-phenyl-(4H)-3, 1-benzoxazi -4 -one (2)}

A mixture of (1) $(0.01 \mathrm{~mol})$ and acetic anhydride $(50 \mathrm{~mL})$ was heated on a water bath for $5 \mathrm{hr}$., the solid formed after removal of excess acetic anhydride was triturated with petroleum ether $40-60^{\circ} \mathrm{C}$ and recrystallized from cyclohexane to give (2).

Colour: white, M.P.: $173-174^{\circ} \mathrm{C}$, Yield: $80 \%$, IR $(\mathrm{KBr})\left(\mathrm{v}, \mathrm{cm}^{-1}\right): 1755(\mathrm{C}=\mathrm{O}), 1607(\mathrm{C}=\mathrm{N}),{ }^{1} \mathrm{H}-\mathrm{NMR}\left(\mathrm{DMSO}-\mathrm{d}_{6}\right): \delta$ (ppm): $8.39(\mathrm{~s}, 1 \mathrm{H}, \mathrm{ArH}), 8.26-8.17(\mathrm{~m}, 3 \mathrm{H}, \mathrm{ArH}), 7.68-7.48(\mathrm{~m}, 4 \mathrm{H}, \mathrm{ArH}) . \mathrm{MS}, \mathrm{m} / \mathrm{z}(\%): 349\left(\mathrm{M}^{+}, 75\right), 305(20), 105$ (100), 77 (100), 51 (35).Anal.Calcd. for $\mathrm{C}_{14} \mathrm{H}_{8} \mathrm{NO}_{2} \mathrm{I}$ (349.12): C, 48.16, H,2.31; N, 4.01. Found: C, 48.36, H, 2.40; N, 4.44.

\subsection{General method for synthesis $(3 \mathrm{a}-\mathrm{g})$ :-}

A mixture of (2) $(0.01 \mathrm{~mol})$ and primary amines, namely 4 - methoxy aniline, benzyl amine, ethyl amine, methyl amine, sec - butyl amine, hydrazine hydrate and phenyl hydrazine $(0.01 \mathrm{~mol})$, in ethanol $(30 \mathrm{~mL})$ was refluxed for $3 \mathrm{hr}$. The solid separated after concentration of ethanol was filtered off, and recrystallized from a suitable solvent to give $(3 \mathbf{a}-\mathbf{g})$.

\subsubsection{N-(4-Methoxy phenyl)- 2 -benzamido- 5- iodobenzamide (3a)}

Colour: white, M.P.: $180-182^{\circ} \mathrm{C}$, Yield: $70 \%$,Recrystalized from: cyclohexane, IR $(\mathrm{KBr})\left(\mathrm{v}, \mathrm{cm}^{-1}\right): 3267,3136(\mathrm{NH}), 1681$, $1648(\mathrm{C}=\mathrm{O}) .{ }^{1} \mathrm{H}-\mathrm{NMR}\left(\mathrm{DMSO}-\mathrm{d}_{6}\right): \delta(\mathrm{ppm}): 11.80(\mathrm{~s}, 1 \mathrm{H}, \mathrm{NH}), 10.49(\mathrm{~s}, 1 \mathrm{H}, \mathrm{NH}), 8.45-8.23(\mathrm{~m}, 3 \mathrm{H}, \mathrm{ArH}), 7.95-7.88$ $(\mathrm{m}, 3 \mathrm{H}, \mathrm{ArH}), 7.62$ - $7.53(\mathrm{~m}, 3 \mathrm{H}, \mathrm{ArH}), 7.37-6.82(\mathrm{~m}, 3 \mathrm{H}, \mathrm{ArH}), 3.70\left(\mathrm{~s}, 3 \mathrm{H}, \mathrm{OCH}_{3}\right) . \mathrm{MS}, \mathrm{m} / \mathrm{z}(\%): 472\left(\mathrm{M}^{+}, 3\right), 349(12)$, 271 (2), 123 (100), 105 (48), 77 (39). Anal.Calcd.For $\mathrm{C}_{21} \mathrm{H}_{17} \mathrm{~N}_{2} \mathrm{O}_{3} \mathrm{l}$ (472.27): C, 53.41, H, 3.63; N, 5.93. Found: C, 53.48, H, $3.67 ; \mathrm{N}, 6.01$.

\subsubsection{N- Benzyl -2-benzamido- 5- iodobenzamide (3b)}

Colour: white, M.P.: $184-185^{\circ} \mathrm{C}$, Yield: $60 \%$, Recrystallized from: ethanol, IR $(\mathrm{KBr})\left(\mathrm{v}, \mathrm{cm}^{-1}\right): 3296(\mathrm{NH}), 1680$, 1623(C=O). ${ }^{1} \mathrm{H}-\mathrm{NMR}\left(\mathrm{DMSO}-\mathrm{d}_{6}\right): \delta(\mathrm{ppm}): 12.41(\mathrm{~s}, 1 \mathrm{H}, \mathrm{NH}), 9.48(\mathrm{br}, 1 \mathrm{H}, \mathrm{NH}), 8.48-8.45(\mathrm{~d}, 2 \mathrm{H}, \mathrm{ArH}), 8.23(\mathrm{~s}, 1 \mathrm{H}$, ArH), 7.93- $7.88(\mathrm{~m}, 3 \mathrm{H}, \mathrm{ArH}), 7.63-7.54(\mathrm{~m}, 3 \mathrm{H}, \mathrm{ArH}), 7.37-7.24(\mathrm{~m}, 4 \mathrm{H}, \mathrm{ArH}), 4.53-4.51\left(\mathrm{~d}, 2 \mathrm{H}, \mathrm{CH}_{2}\right) . \mathrm{MS}, \mathrm{m} / \mathrm{z}(\%):$ $456(\mathrm{M}+, 9), 447(14), 418(16), 386$ (14), 205 (15), 117 (18), 55 (100). Anal.Calcd.For $\mathrm{C}_{21} \mathrm{H}_{17} \mathrm{~N}_{2} \mathrm{O}_{2} \mathrm{l}(456.27): \mathrm{C}, 55.28, \mathrm{H}$, $3.75 ; \mathrm{N}, 6.14$. Found: C, 54.99, H, 3.79; N, 6.20 .

\subsubsection{N-Ethyl-2- benzamido- 5 -iodobenzamide (3c)}

Colour: white, M.P.: $109-111^{\circ} \mathrm{C}$, Yield: $80 \%$, Recrystallized from: ethanol, IR $(\mathrm{KBr})\left(\mathrm{v}, \mathrm{cm}^{-1}\right): 3280(\mathrm{NH}), 1683$, 1628(C=O). ${ }^{1} \mathrm{H}-\mathrm{NMR}\left(\mathrm{DMSO}-\mathrm{d}_{6}\right): \delta(\mathrm{ppm}): 12.57(\mathrm{~s}, 1 \mathrm{H}, \mathrm{NH}), 9.10(\mathrm{br}, 1 \mathrm{H}, \mathrm{NH}), 8.48-8.45(\mathrm{~d}, 1 \mathrm{H}, \mathrm{ArH}), 8.16(\mathrm{~s}, 1 \mathrm{H}$, ArH), 7.93- $7.87(\mathrm{~m}, 3 \mathrm{H}, \mathrm{ArH}), 7.64-7.59(\mathrm{~m}, 3 \mathrm{H}, \mathrm{ArH}), 3.38-3.34\left(\mathrm{q}, 2 \mathrm{H}, \mathrm{CH}_{2}\right), 1.17-1.13\left(\mathrm{t}, 3 \mathrm{H}, \mathrm{CH}_{3}\right) . \mathrm{MS}, \mathrm{m} / \mathrm{z}(\%)$ : $394\left(\mathrm{M}^{+}, 20\right), 376(39), 245$ (32), 105 (100). Anal.Calcd.For $\mathrm{C}_{16} \mathrm{H}_{15} \mathrm{~N}_{2} \mathrm{O}_{2} \mathrm{I}$ (394.21): C, 48.75, H, 3.83; N, 7.11. Found: C, 49.01, H, 3.88; N, 6.98 .

\subsubsection{N-Methyl- 2 -benzamido -5- iodobenzamide (3d)}

Colour: white, M.P.: $188-190^{\circ} \mathrm{C}$, Yield: $70 \%$, Recrystallized from: ethanol, IR $(\mathrm{KBr})\left(\mathrm{v}, \mathrm{cm}^{-1}\right): 3317(\mathrm{NH}), 1680$, $1627(\mathrm{C}=\mathrm{O}) .{ }^{1} \mathrm{H}-\mathrm{NMR}\left(\mathrm{DMSO}-\mathrm{d}_{6}\right): \delta(\mathrm{ppm}): 12.63(\mathrm{~s}, 1 \mathrm{H}, \mathrm{NH}), 9.01(\mathrm{br}, 1 \mathrm{H}, \mathrm{NH}), 8.49-8.47(\mathrm{~d}, 1 \mathrm{H}, \mathrm{ArH}), 8.16(\mathrm{~s}, 1 \mathrm{H}$, ArH), $7.94-7.88(\mathrm{~m}, 3 \mathrm{H}, \mathrm{ArH}), 7.64-7.57(\mathrm{~m}, 3 \mathrm{H}, \mathrm{ArH}), 2.82-2.80\left(\mathrm{~d}, 3 \mathrm{H}, \mathrm{CH}_{3}\right) . \mathrm{MS}, \mathrm{m} / \mathrm{z}(\%): 379\left(\mathrm{M}^{+}-1\right)(2), 348(4)$, 247 (2), 110 (1.5), 80 (100), 64 (50). Anal.Cacld.For $\mathrm{C}_{15} \mathrm{H}_{13} \mathrm{~N}_{2} \mathrm{O}_{2} \mathrm{I}(380.18)$ : C, 47.39, H, 3.44; N, 7.37. Found: C, 47.42, H, $3.50 ; \mathrm{N}, 7.42$.

\subsubsection{N-Sec. Butyl -2-benzamido- 5- iodobenzamide (3e)}

Colour: white, M.P.: $146-148^{\circ} \mathrm{C}$, Yield: $70 \%$, Recrystallized from: ethanol, IR $(\mathrm{KBr})\left(\mathrm{v}, \mathrm{cm}^{-1}\right): 3310(\mathrm{NH}), 1681$, 1625(C=O). ${ }^{1} \mathrm{H}-\mathrm{NMR}\left(\mathrm{DMSO}-\mathrm{d}_{6}\right): \delta(\mathrm{ppm}): 12.48(\mathrm{~s}, 1 \mathrm{H}, \mathrm{NH}), 8.80(\mathrm{~d}, 1 \mathrm{H}, \mathrm{NH}), 8.46-8.43(\mathrm{~d}, 1 \mathrm{H}, \mathrm{ArH}), 8.16(\mathrm{~S}, 1 \mathrm{H}$ $\mathrm{ArH}), 7.93-7.87(\mathrm{~m}, 3 \mathrm{H}, \mathrm{ArH}), 7.64-7.59(\mathrm{~m}, 3 \mathrm{H}, \mathrm{ArH}), 4.10-3.95(\mathrm{~m}, 1 \mathrm{H}, \mathrm{CH}), 1.57-1.53\left(\mathrm{~m}, 2 \mathrm{H}, \mathrm{CH}_{2}\right), 1.16-1.14(\mathrm{~d}$, 
$\left.3 \mathrm{H}, \mathrm{CH}_{3}\right), 0.90-0.85$ (t, 3H, CH ). MS, m/z (\%): 422(M+, 6.5), 350 (15), 268 (12), 148 (33), 105 (100), 77 (43). Anal.Calcd.For $\mathrm{C}_{18} \mathrm{H}_{19} \mathrm{~N}_{2} \mathrm{O}_{2} \mathrm{I}$ (422.26): C, 51.20, H, 4.53; N, 6.63. Found: C, 51.30, H, 4.53; N, 6.66.

\subsubsection{2-Benzamido-5-iodobenzoylhydrazide (3f)}

Colour: white, M.P.: $204-206^{\circ} \mathrm{C}$, Yield: $70 \%$,Recrystallized from ethanol, IR $(\mathrm{KBr})\left(\mathrm{v}, \mathrm{cm}^{-1}\right): 3300,3250(\mathrm{NH}), 1688$, $1640(\mathrm{C}=\mathrm{O}) .{ }^{1} \mathrm{H}-\mathrm{NMR}\left(\mathrm{DMSO}-\mathrm{d}_{6}\right): \delta(\mathrm{ppm}): 12.45(\mathrm{br}, 1 \mathrm{H}, \mathrm{NH}), 8.48-8.46(\mathrm{~d}, 1 \mathrm{H}, \mathrm{ArH}), 8.10(\mathrm{~s}, 1 \mathrm{H}, \mathrm{ArH}), 7.95-7.88$ $(\mathrm{m}, 3 \mathrm{H}, \mathrm{ArH}), 7.78-7.40(\mathrm{~m}, 3 \mathrm{H}, \mathrm{ArH}), 5.67(\mathrm{~s}, 1 \mathrm{H}, \mathrm{NH}), 4.77\left(\mathrm{br}, 2 \mathrm{H}, \mathrm{NH}_{2}\right) . \mathrm{MS}, \mathrm{m} / \mathrm{z}(\%): 381\left(\mathrm{M}^{+}, 23\right), 287(38), 237(26)$, 173 (23), 127 (31), 77 (52), 55 (100). Anal.Calcd.For $\mathrm{C}_{14} \mathrm{H}_{12} \mathrm{~N}_{3} \mathrm{O}_{2} \mathrm{I}$ (381.17): C, 44.11, H, 3.17; N, 11.02. Found: C, 44.18, H, 2.99; N, 10.98 .

\subsubsection{N-Phenyl - 2- benzamido -5 - iodo-benzoylhydrazide (3g)}

Colour: white, M.P.: $250-252^{\circ} \mathrm{C}$, Yield: $60 \%$, Recrystallized from toluene, IR $(\mathrm{KBr})\left(\mathrm{v}, \mathrm{cm}^{-1}\right): 3325,3188(\mathrm{NH}), 1664$, 1646(C=O). ${ }^{1} \mathrm{H}-\mathrm{NMR}\left(\mathrm{DMSO}-\mathrm{d}_{6}\right): \delta(\mathrm{ppm}): 12.03(\mathrm{~s}, 1 \mathrm{H}, \mathrm{NH}), 10.75-10.74(\mathrm{~d}, 1 \mathrm{H}, \mathrm{NH}), 8.44-8.41(\mathrm{~d}, 1 \mathrm{H}, \mathrm{NH})$, 8.31(s, 1H, ArH), $7.99-7.87(\mathrm{~m}, 4 \mathrm{H}, \mathrm{ArH}), 7.62-7.52(\mathrm{~m}, 3 \mathrm{H}, \mathrm{ArH}), 7.16-7.13(\mathrm{~m}, 2 \mathrm{H}, \mathrm{ArH}), 6.85-6.72(\mathrm{~m}, 3 \mathrm{H}, \mathrm{ArH})$. MS, m/z (\%): 457(M+, 1), 439 (4), 348 (46), 245 (40), 105 (50), 80 (95), 64 (100). Anal.Calcd. For $\mathrm{C}_{20} \mathrm{H}_{16} \mathrm{~N}_{3} \mathrm{O}_{2} \mathrm{I}(457.26)$ : C, 52.53, H, 3.52; N, 9.19. Found: C, 52.60, H, 3.51; N, 9.21.

\subsection{General method for synthesis $(4 a-c)$ :-}

A mixture of (2) $(0.01 \mathrm{~mol})$ and secondary amines, namely morpholine, piperidine and /or piperazine $(0.01 \mathrm{~mol})$ in $1,4-$ dioxane $(30 \mathrm{~mL})$ was refluxed for $5 \mathrm{hr}$. The solid separated after concentration of 1,4-dioxane was filtered off, and recrystallized from a suitable solvent to give $(4 a-c)$.

\subsubsection{N-(4-lodo-2(morpholine -1-carbonyl) phenylbenzamide (4a)}

Colour: colorless, M.P.: $204-206{ }^{\circ} \mathrm{C}$, Yield: 55\%, Recrystallized from toluene/ethanol, IR $(\mathrm{KBr})\left(\mathrm{v}, \mathrm{cm}^{-1}\right): 3196,3100$ $(\mathrm{NH}), 1656(\mathrm{C}=\mathrm{O}) .{ }^{1} \mathrm{H}-\mathrm{NMR}\left(\mathrm{DMSO}-\mathrm{d}_{6}\right): \delta(\mathrm{ppm}): 10.25(\mathrm{~s}, 1 \mathrm{H}, \mathrm{NH}), 7.92-7.90(\mathrm{~d}, 1 \mathrm{H}, \mathrm{ArH}), 7.83-7.40(\mathrm{~m}, 7 \mathrm{H}, \mathrm{ArH})$, 3.58 - $3.55\left(\mathrm{~m}, 8 \mathrm{H},\left(\mathrm{CH}_{2}\right)_{4}\right)$. MS, m/z (\%): 434(M+ -2, 13), 408 (19), 350 (25), 272 (16), 213 (14), 105 (92), 77 (100). Anal.Calcd. For $\mathrm{C}_{18} \mathrm{H}_{17} \mathrm{~N}_{2} \mathrm{O}_{3} \mathrm{I}$ (436.24): C, 49.56, H, 3.92; N, 6.42. Found: C, 49.60, H, 3.95; N, 6.45.

\subsubsection{N-(4-lodo-2(piperidine-1- carbonyl) phenylbenzamide (4b)}

Colour: white, M.P.: $149-151{ }^{\circ} \mathrm{C}$, Yield: $50 \%$, Recrystallized from ethanol, IR $(\mathrm{KBr})\left(\mathrm{v}, \mathrm{cm}^{-1}\right): 3287(\mathrm{NH}), 1672(\mathrm{C}=\mathrm{O}) .{ }^{1} \mathrm{H}-$ NMR (DMSO - $\left.\mathrm{d}_{6}\right): \delta(\mathrm{ppm}): 10.14(\mathrm{br}, 1 \mathrm{H}, \mathrm{NH}), 7.88(\mathrm{~S}, 1 \mathrm{H}, \mathrm{ArH}), 7.87(\mathrm{~s}, 1 \mathrm{H}, \mathrm{ArH}), 7.79(\mathrm{~d}, 1 \mathrm{H}, \operatorname{ArH}), 7.64(\mathrm{~d}, 1 \mathrm{H}, \operatorname{ArH})$, $7.60-7.50(\mathrm{~m}, 3 \mathrm{H}, \mathrm{ArH}), 7.43(\mathrm{~d}, 1 \mathrm{H}, \mathrm{ArH}), 3.63-3.47\left(\mathrm{~m}, 4 \mathrm{H}, \mathrm{N}\left(\mathrm{CH}_{2}\right)_{2}\right), 1.52-1.47\left(\mathrm{~m}, 6 \mathrm{H},\left(\mathrm{CH}_{2}\right)_{3}\right) . \mathrm{MS}, \mathrm{m} / \mathrm{z}(\%):$ 434( $\left.\mathrm{M}^{+}, 13\right), 372$ (16), 270(15), 105(92), 77 (100). Anal.Calcd. For $\mathrm{C}_{19} \mathrm{H}_{19} \mathrm{~N}_{2} \mathrm{O}_{2} \mathrm{l}$ (434.27): C, 52.54, $\mathrm{H}, 4.41 ; \mathrm{N}, 6.45$. Found: C, 52.58, H, 4.40; N, 6.50 .

\subsubsection{N-(4-lodo- 2 (piperazine -1-carbonyl) phenylbenzamide (4c)}

Colour: white, M.P.: $126-128{ }^{\circ} \mathrm{C}$, Yield: $65 \%$, Recrystallized from toluene, IR $(\mathrm{KBr})\left(\mathrm{v}, \mathrm{cm}^{-1}\right): 3250(\mathrm{NH}), 1671(\mathrm{C}=\mathrm{O}) .{ }^{1} \mathrm{H}-$ NMR (DMSO - $\left.\mathrm{d}_{6}\right): \delta(\mathrm{ppm}): 11.75(\mathrm{~s}, 1 \mathrm{H}, \mathrm{NH}), 8.03(\mathrm{~d}, 2 \mathrm{H}, \mathrm{ArH}), 7.70-7.52(\mathrm{~m}, 6 \mathrm{H}, \mathrm{ArH}), 5.10(\mathrm{~s}, 1 \mathrm{H}, \mathrm{NH}), 2.67\left(\mathrm{~s}, 8 \mathrm{H},\left(\mathrm{CH}_{2}\right) 4\right) \cdot \mathrm{MS}$, $\mathrm{m} / \mathrm{z}(\%):$ 434(M $\left.{ }^{+}-1,29\right), 360$ (28), 268 (41), 177(30), 105 (100), 77(71). Anal.Calcd. For $\mathrm{C}_{18} \mathrm{H}_{18} \mathrm{~N}_{3} \mathrm{O}_{2} \mathrm{l}$ (435.26): C, 49.67, $\mathrm{H}, 4.17 ; \mathrm{N}, 9.65$. Found: C, 49.88, H, 4.22; N, 9.80 .

\subsection{General method for synthesis $(5 a-b):-$}

A mixture of (2) $(0.01 \mathrm{~mol})$, ethyl alcohol and / or $\mathrm{n}-$ butyl alcohol $(50 \mathrm{~mL})$ in pyridine $(1 \mathrm{~mL})$ was refluxed for $4 \mathrm{hr}$. The solid formed after concentration of the solvent was collected and recrystallized from a suitable solvent to give (5a) \& (5b) respectively.

\subsubsection{Ethyl- 5-iodo- 2-benzamidobenzoate (5a)}

Colorless crystals, M.P.: $282-284^{\circ} \mathrm{C}$, Yield: $70 \%$, Recrystallized from benzene/ toluene, IR $(\mathrm{KBr})\left(\mathrm{v}, \mathrm{cm}^{-1}\right): 3247(\mathrm{NH})$, 1690, $1669(\mathrm{C}=\mathrm{O}) .{ }^{1} \mathrm{H}-\mathrm{NMR}\left(\mathrm{DMSO}-\mathrm{d}_{6}\right): \delta(\mathrm{ppm}): 11.48(\mathrm{~s}, 1 \mathrm{H}, \mathrm{NH}), 8.35(\mathrm{~d}, 1 \mathrm{H}, \mathrm{ArH}), 8.32(\mathrm{~d}, 1 \mathrm{H}, \mathrm{ArH}), 8.25-8.01$ $(\mathrm{m}, 3 \mathrm{H}, \mathrm{ArH}), 7.99-7.59(\mathrm{~m}, 3 \mathrm{H}, \mathrm{ArH}), 4.36-4.33\left(\mathrm{q}, 2 \mathrm{H}, \mathrm{CH}_{2}\right), 1.34-1.29\left(\mathrm{t}, 3 \mathrm{H}, \mathrm{CH}_{3}\right) . \mathrm{MS}, \mathrm{m} / \mathrm{z}(\%): 395\left(\mathrm{M}^{+}, 22\right)$, 105(100), 77(50), 51 (18). Anal.Calcd. For $\mathrm{C}_{16} \mathrm{H}_{14} \mathrm{NO}_{3} \mathrm{I}$ (395.19): C, 48.63, H, 3.57; N, 3.54. Found: $\mathrm{C}, 48.88, \mathrm{H}, 3.60 ; \mathrm{N}$, 3.59 .

\subsection{2 n-Butyl -5-iodo -2-benzamidobenzoate (5b)}

Colorless crystals, M.P.: $280-282^{\circ} \mathrm{C}$, Yield: $77 \%$, Recrystallized from ethanol, IR $(\mathrm{KBr})\left(\mathrm{v}, \mathrm{cm}^{-1}\right): 3243(\mathrm{NH}), 1687,1668$ $(\mathrm{C}=\mathrm{O}) .{ }^{1} \mathrm{H}-\mathrm{NMR}\left(\mathrm{DMSO}-\mathrm{d}_{6}\right): \delta(\mathrm{ppm}): 11.47(\mathrm{~s}, 1 \mathrm{H}, \mathrm{NH}), 8.35-8.32(\mathrm{~d}, 1 \mathrm{H}, \mathrm{ArH}), 8.23(\mathrm{~s}, 1 \mathrm{H}, \mathrm{ArH}), 8.01-7.93(\mathrm{~m}, 3 \mathrm{H}$, ArH), $7.68-7.56(\mathrm{~m}, 3 \mathrm{H}, \mathrm{ArH}), 4.32-4.28\left(\mathrm{t}, 2 \mathrm{H}, \mathrm{CH}_{2}\right), 1.73-1.64\left(\mathrm{~m}, 2 \mathrm{H}, \mathrm{CH}_{2}\right), 1.45-1.32\left(\mathrm{~m}, 2 \mathrm{H}, \mathrm{CH}_{2}\right), 0.92-0.88$ (t, $\left.3 \mathrm{H}, \mathrm{CH}_{3}\right) . \mathrm{MS}, \mathrm{m} / \mathrm{z}(\%): 423\left(\mathrm{M}^{+}, 12\right), 149(5), 105$ (100), 77(25), 56 (70). Anal.Calcd. For $\mathrm{C}_{18} \mathrm{H}_{18} \mathrm{NO}_{3} \mathrm{l}(423.24): \mathrm{C}, 51.08$, H, 4.29; N, 3.31. Found: C, 51.22, H, 4.33; N, 3.38.

\subsection{General method for synthesis (6a-b):-}

A mixture of (2) $(0.01 \mathrm{~mol})$, hydrazine hydrate and/or hydroxylamine hydrochloride $(0.01 \mathrm{~mol})$ in $\mathrm{n}-\mathrm{butanol}(50 \mathrm{~mL}) \mathrm{was}$ refluxed for $5 \mathrm{hr}$. The solid formed after concentration of solvent was collected by filtration and recrystallized from a suitable solvent to give $(6 a) \&(6 b)$ respectively. 


\subsubsection{3-Amino- 6-iodo- 2-phenylquinazolin- 4-one (6a)}

Colour: white, M.P.: $146-148^{\circ} \mathrm{C}$, Yield: $75 \%$, Recrystallized from toluene/ethanol. IR $(\mathrm{KBr})\left(\mathrm{v}, \mathrm{cm}^{-1}\right): 3310(\mathrm{NH}), 1665$ $(\mathrm{C}=\mathrm{O}) .{ }^{1} \mathrm{H}-\mathrm{NMR}\left(\mathrm{DMSO}-\mathrm{d}_{6}\right): \delta(\mathrm{ppm}): 8.46-8.45(\mathrm{~d}, 1 \mathrm{H}, \mathrm{ArH}), 8.13-8.12(\mathrm{~d}, 1 \mathrm{H}, \mathrm{ArH}), 7.82-7.79(\mathrm{~m}, 2 \mathrm{H}, \mathrm{ArH}), 7.52$ - $7.44(\mathrm{~m}, 4 \mathrm{H}, \mathrm{ArH}), 5.67$ (s, 2H, NH ). MS, m/z (\%): $363\left(\mathrm{M}^{+}, 2\right), 349$ (9), 285 (2), 105 (100), 77 (49). Anal.Calcd. for $\mathrm{C}_{14} \mathrm{H}_{10} \mathrm{~N}_{3} \mathrm{Ol}$ (363.15): C, 46.30, H, 2.77; N, 11.57. Found: C, 46.48, H, 2.80; N, 11.62.

\subsubsection{3-Hydroxy- 6 -iodo-2-phenylquinazolin-4-one (6b)}

Colour: white, M.P.: $210-212^{\circ} \mathrm{C}$, Yield: $55 \%$, Recrystallized from ethanol. IR $(\mathrm{KBr})\left(\mathrm{v}, \mathrm{cm}^{-1}\right): 3432(\mathrm{OH}), 1661(\mathrm{C}=\mathrm{O}) .{ }^{1} \mathrm{H}-$ NMR (DMSO - $\left.\mathrm{d}_{6}\right): \delta(\mathrm{ppm}): 11.75(\mathrm{~s}, 1 \mathrm{H}, \mathrm{OH}), 8.52-8.50(\mathrm{~d}, 1 \mathrm{H}, \mathrm{ArH}), 8.30(\mathrm{~s}, 1 \mathrm{H}, \mathrm{ArH}), 7.96-7.83(\mathrm{~m}, 3 \mathrm{H}, \mathrm{ArH}), 7.61$ - 7.52 (m, 3H, ArH). MS, m/z (\%): 364 (M $\left.{ }^{+}, 85\right), 334$ (37), 245 (24), 178 (17), 105 (71), 77 (100). Anal.Calcd. for $\mathrm{C}_{14} \mathrm{H}_{9} \mathrm{~N}_{2} \mathrm{O}_{2} \mathrm{I}(364.14): \mathrm{C}, 46.18, \mathrm{H}, 2.49 ; \mathrm{N}, 7.69$. Found: C, 46.23, H, 2.52; N, 7.70.

\subsection{6-lodo-2-phenylquinazolin- 4-one (7)}

A mixture of (2) $(0.01 \mathrm{~mol})$ and ammonium acetate $(0.04 \mathrm{~mol})$ was heated in neat for $3 \mathrm{hr}$ at $170^{\circ} \mathrm{C}$. After cooling, the reaction mixture was triturated with hot water, filteredoff, dried and recrystallized from ethanol to give (7).

Colour: white, M.P.: $216-218^{\circ} \mathrm{C}$, Yield: 45\%. IR (KBr) $\left(\mathrm{v}, \mathrm{cm}^{-1}\right): 3384,3206(\mathrm{NH}), 1665(\mathrm{C}=\mathrm{O}) .{ }^{1} \mathrm{H}-\mathrm{NMR}\left(\mathrm{DMSO}-\mathrm{d}_{6}\right): \delta$ (ppm): $8.39(\mathrm{~d}, 1 \mathrm{H}, \mathrm{ArH}), 8.24(\mathrm{~d}, 1 \mathrm{H}, \mathrm{ArH}), 8.23(\mathrm{~d}, 1 \mathrm{H}, \mathrm{ArH}), 8.19-8.17(\mathrm{~m}, 2 \mathrm{H}, \mathrm{ArH}, \mathrm{NH}), 7.68-7.58(\mathrm{~m}, 3 \mathrm{H}, \mathrm{ArH}), 7.5$ (d, $1 \mathrm{H}, \mathrm{ArH}$ ).Anal.Calcd. for $\mathrm{C}_{14} \mathrm{H}_{9} \mathrm{~N}_{2} \mathrm{Ol}$ (348.14): C, 48.30, H, 2.60; N, 8.05. Found: C, 48.38, H, 2.57; N, 7.99.

\subsection{General method for synthesis $(8 a-b):-$}

A mixture of (2) $(0.01 \mathrm{~mol})$, semicarbazide hydrochloride and /or thiosemicarbazide $(0.01 \mathrm{~mol})$ in pyridine $(30 \mathrm{~mL})$ was refluxed for $5 \mathrm{hr}$. After cooling, the reaction mixture was poured onto ice cold hydrochloric acid, the precipitate formed was collected, dried and recrystallized from a suitable solvent to give $(\mathbf{8 a}) \&(\mathbf{8 b})$ respectively.

\subsubsection{4-lodo-N-(2 - (5-oxo-4, 5-dihydro-1H -1, 2, 4 -triazol -3-yl) phenyl benzamide (8a)}

Colour: white, M.P.: $295-297^{\circ} \mathrm{C}$, Yield: 55\%, Recrystallized from methanol. IR $(\mathrm{KBr})\left(\mathrm{v}, \mathrm{cm}^{-1}\right): 3321,3278(\mathrm{NH}), 1667$ $(\mathrm{C}=\mathrm{O}) .{ }^{1} \mathrm{H}-\mathrm{NMR}\left(\mathrm{DMSO}-\mathrm{d}_{6}\right): \delta(\mathrm{ppm}): 10.54(\mathrm{~s}, 1 \mathrm{H}, \mathrm{NH}), 8.45(\mathrm{~d}, 1 \mathrm{H}, \mathrm{ArH}), 8.16-8.12(\mathrm{~m}, 2 \mathrm{H}, \mathrm{ArH}), 7.97-7.45(\mathrm{~m}$, $5 \mathrm{H}, \mathrm{ArH}), 5.68$ (s, 2H, $2 \mathrm{NH}) . \mathrm{MS}, \mathrm{m} / \mathrm{z}(\%): 406\left(\mathrm{M}^{+}, 26\right), 388$ (43), 260 (40), 221 (44), 137 (42), 105 (67), 75 (100). Anal.Calcd. For $\mathrm{C}_{15} \mathrm{H}_{11} \mathrm{~N}_{4} \mathrm{O}_{2} \mathrm{I}$ (406.18): C, 44.35, H, 2.73; N, 13.79. Found: C, 44.40, H, 2.75; N, 13.82

\subsubsection{4-lodo-N-(2-(5-thioxo-4, 5-dihydro -1H-1, 2, 4-triazol-3-yl) phenyl benzamide (8b)}

Colour: green, M.P.: $209-212^{\circ} \mathrm{C}$, Yield: $65 \%$, Recrystallized from methanol /ethanol. IR $(\mathrm{KBr})\left(\mathrm{v}, \mathrm{cm}^{-1}\right): 3111(\mathrm{NH}), 1653$ $(\mathrm{C}=\mathrm{O}) .{ }^{1} \mathrm{H}-\mathrm{NMR}\left(\mathrm{DMSO}-\mathrm{d}_{6}\right): \delta(\mathrm{ppm}): 11.70(\mathrm{~s}, 1 \mathrm{H}, \mathrm{NH}), 8.53(\mathrm{~d}, 1 \mathrm{H}, \mathrm{ArH}), 8.30(\mathrm{~d}, 1 \mathrm{H}, \mathrm{ArH}), 7.99-7.92(\mathrm{~m}, 3 \mathrm{H}, \mathrm{ArH})$, $7.66-7.56(\mathrm{~m}, 3 \mathrm{H}, \mathrm{ArH}), 5.70$ (s, 2H, $2 \mathrm{NH}) . \mathrm{MS}, \mathrm{m} / \mathrm{z}(\%): 422\left(\mathrm{M}^{+}, 60\right), 380$ (28), 341 (47), 280 (29), 179 (53), 80 (95), 64 (100). Anal.Calcd. For $\mathrm{C}_{15} \mathrm{H}_{11} \mathrm{~N}_{4} \mathrm{OSI}$ (422.24): C, 42.67, H, 2.62,N, 13.27; S,7.59. Found: C, 42.71, H, 2.66, N, 13.29; S, 7.62 .

\subsection{General method for synthesis (9) \& (10):-}

A mixture of (2) $(0.01 \mathrm{~mol})$ and sodium azide $(0.01 \mathrm{~mol})$, in acetic acid $(50 \mathrm{~mL})$ was refluxed for $5 \mathrm{hr}$. After cooling, the reaction mixture was poured onto water; the precipitate formed was collected, dried and fractionally recrystallized from benzene to give (9), while the insoluble part was crystallized from ethanol to give (10).

\subsubsection{5-lodo-2-(5-phenyl- tetrazol-1-yl) benzoic acid (9)}

Colour: white, M.P.: $169-170^{\circ} \mathrm{C}$, Yield: $40 \%$. IR $(\mathrm{KBr})\left(\mathrm{v}, \mathrm{cm}^{-1}\right): 3414(\mathrm{OH}), 1697(\mathrm{C}=\mathrm{O}) .{ }^{1} \mathrm{H}-\mathrm{NMR}\left(\mathrm{DMSO}-\mathrm{d}_{6}\right): \delta(\mathrm{ppm})$ : $12.15(\mathrm{~s}, 1 \mathrm{H}, \mathrm{OH}), 8.53(\mathrm{~d}, 1 \mathrm{H}, \mathrm{ArH}), 8.30(\mathrm{~d}, 1 \mathrm{H}, \mathrm{ArH}), 7.96-7.93(\mathrm{~m}, 3 \mathrm{H}, \mathrm{ArH}), 7.74-7.55(\mathrm{~m}, 3 \mathrm{H}, \mathrm{ArH}) . \mathrm{MS}, \mathrm{m} / \mathrm{z}(\%)$ : $392\left(\mathrm{M}^{+}, 23\right), 349$ (85), 284 (27), 233 (30), 162 (27), 77 (100).Anal. Calcd. For $\mathrm{C}_{14} \mathrm{H}_{9} \mathrm{~N}_{4} \mathrm{O}_{2} \mathrm{l}(392.15): \mathrm{C}, 42.88, \mathrm{H}, 2.31 ; \mathrm{N}$ 14.29. Found: $\mathrm{C}, 42.95, \mathrm{H}, 2.55 ; \mathrm{N}, 14.33$.

\subsubsection{1-Benzoyl-5-iodo -1, 3-dihydro-benzoimidazol-2- one (10)}

Colour: white, M.P.: $245-247^{\circ} \mathrm{C}$, Yield: $35 \%$. IR (KBr) $\left(\mathrm{v}, \mathrm{cm}^{-1}\right): 3337,3115(\mathrm{NH}), 1729,1662(\mathrm{C}=\mathrm{O}) .{ }^{1} \mathrm{H}-\mathrm{NMR}(\mathrm{DMSO}-$ $\left.\mathrm{d}_{6}\right): \delta(\mathrm{ppm}): 11.40(\mathrm{~s}, 1 \mathrm{H}, \mathrm{NH}), 8.50(\mathrm{~d}, 1 \mathrm{H}, \mathrm{ArH}), 8.29(\mathrm{~d}, 1 \mathrm{H}, \mathrm{ArH}), 7.96-7.92(\mathrm{~m}, 3 \mathrm{H}, \mathrm{ArH}), 7.74-7.54(\mathrm{~m}, 3 \mathrm{H}, \mathrm{ArH})$. MS, m/z (\%): $364\left(\mathrm{M}^{+}, 24\right), 331$ (22), 274 (29), 194(9), 142 (28), 77 (100).Anal. Calcd. for $\mathrm{C}_{14} \mathrm{H}_{9} \mathrm{~N}_{2} \mathrm{O}_{2} \mathrm{l}(364.14)$ : C, 46.18, H, 2.49; N, 7.69. Found: C, 46.23, H, 2.52;N,7.72.

\section{REFERENCES}

[1] Erusalimsky ,J. and Franklin, R.; Experimental Hematology, (2002),30, 625-626 .

[2] Farghaly ,A.M., Soliman ,R., Khalil ,M.A., Bekhit A.A., and El-Din ,A.; BollettinoChimicoFarmaceutico, (2002),141, 372-378

[3] Blackburn,C., LaMorche, M. J. Brown, Bioorg. \& Med. Chem. Lett. (2006),16(10), 2621.

[4] El-Helby ,A.; Egypt Phar. Sci. (1995),36, 287. 
[5] El-Khamry ,A.A., El-Nagdy, S., Habashy ,M.M., and El-Bassiouny, F.A. Pharmazie, (1989),44, 312.

[6] Ossman ,A.E. and Barakat, S.E., Sauidi Pharm. J. (1994),2, 21.

[7] Madkour ,H.M., Shiba ,S.A., Sayed ,H.M. and Hamed ,A.A., Sulfur Letters, (2001), 24, 151.

[8] Alyaa ,A.S., AbdelMomen, A.E., Sayed ,A.S., and Abdelaal, A.A. Archiv.Der Pharmazie, (2000),333(11),365371.

[9] Al-Obaid,A.M., Abdel-Hamide S.G., El-Kashef, H.A., Abdel-Aziz A.M. and El-Azab A.S.; European Journal of medicinal chemistry (2009),44,2379-2391.

[10] Aly,M.M., Mohamed,Y.A. and El-Bayouki,K.M.; European Journal of medicinal chemistry (2010),45, 3365-3373.

[11] Chandrika,P.M., Yakaiah, T., RamRao ,A.R. and Rao, J.V.; European Journal of medicinal chemistry(2008), 43, 846-852.

[12] Patel,N.B., Patel,J.C. and Barat,G.G.; Orbital Elec. J.Chem., Campo Grand, (2010.)2(3):248-262.

[13] Prajapati,N.K.,Shah,R.M. and Patel,P.S.; Journal of chemical and pharmaceutical research , (2012).,4(5):25742576.

[14] Kaniskan ,N., Kokten ,S. and Celik, I. ;Arkivok (2012), (viii)198-213.

[15] Salih ,N.A.;Journal of Al-Nahrain university (2008),11(2),August,pp.16-23.

[16] Grover ,G. and Kini ,S.G.; European Journal of medicinal chemistry, (2006),41,256-262.

[17] Osborne ,A.G. and Goolamali, Z.; Spectrochimica Acta Part A (2000), 56,1079-1100.

[18] El-Khamry ,A. A., Shiba ,S.A., Shalaby A.A. and Abdalaha, A. A.;

J. Heterocyclic chem.(2006),43, 1189-1193.

[19] Abdalha, A. A., AbouEl.Regal ,M. K., El Kassaby M. A. and Ali, A. T., Synthetic Communications, (2011), 41:2811-2821.

[20] Hamed,A.A., El-Ziaty ,A.K., Abdalha, A. A., Shiba, S. A. and Abdul-Hafeedh,A,A.; European Journal of chemistry (2012), 3(1) 65-70.

[21] Hamed,A.A., El-Ziaty ,A.K., Abdalha, A. A., Shiba, S. A. and Abdul-Hafeedh,A,A.;; World Applied Sciences Journal (2012),19(5):688-698.

[22] Eissa ,A.M and Grasas ,Y.,Aceites, (2007),58(4),379-389. Grayer ,R.J. and Harbone , J.B., Phtochemistry, 37, 1942(1994). 